\title{
Research Article \\ Effect of Electrohydraulic Discharge on Viscosity of Human Blood
}

\author{
G. M. El-Aragi \\ Plasma Physics and Nuclear Fusion Department, Nuclear Research Center, AEA, Cairo 13759, Egypt \\ Correspondence should be addressed to G. M. El-Aragi; elaragi@gmail.com
}

Received 3 April 2013; Revised 15 May 2013; Accepted 29 May 2013

Academic Editor: Ali Hussain Reshak

Copyright (C) 2013 G. M. El-Aragi. This is an open access article distributed under the Creative Commons Attribution License, which permits unrestricted use, distribution, and reproduction in any medium, provided the original work is properly cited.

Electrohydraulic plasma discharge is a novel technology with high efficiency and high speed and can generate chemically active species like free radicals, ions, atoms, and metastables, accompanied by ultraviolet light emission and shock pressure waves. The aim of this work is to examine the effect of electrohydraulic discharge (EHD) system on viscosity of the human blood after different exposure time. The voltage pulsation introduces electric field and temperature jump and at the same time leads to haemolysis of the blood cells. The ratio of blood viscosity under the influence of magnetic field $\eta_{\text {mag }}$ to the viscosity in the absence of magnetic field $\eta$ is directly proportional to the applied magnetic field $H$.

\section{Introduction}

Blood viscosity is one measurement currently obtained invasively via a blood sample and can be defined as the intrinsic resistance to blood flow due to internal friction arising between blood's molecular and particulate components. The viscosity of any fluid (measured in millipascals-seconds) is a function of its sheer stress (force per unit area applied to a fluid layer producing this layer's movement relative to an adjacent fluid layer) and its sheer rate (velocity gradient between two adjacent fluid layers), defined in

$$
(\text { dynamic viscosity }) \eta(\mathrm{mPa} \cdot \mathrm{s})=\frac{\text { shear stress }}{\text { shear rate }} \text {. }
$$

Of the factors influencing blood viscosity, the major contributors include blood plasma, plasma proteins, and both leukocyte and erythrocyte volume (hematocrit), shape, and aggregation [1].

Blood viscosity variations by erythrocytic factors are indicative of various human ailments. The two major components of RBCs resulting in abnormal viscosity measurements are individual $\mathrm{RBC}$ deformation and collective RBC aggregation. Compared to other components of whole blood, the $\mathrm{RBC}$ component is a strong magnetic material, whose orientation has been shown to be affected by external magnetic fields [2]. The magnetic force felt by erythrocytes depending on their magnetic state has been approximated by [3]

$$
F_{\mathrm{BC}}=0.5 \mu_{0} \chi V_{\mathrm{BC}} \nabla(H \cdot H),
$$

where $\mu_{0}$ is the permeability of free space, $\chi$ is the difference in magnetic susceptibility between blood cells and buffer solution (in our case, mainly plasma and white blood cells), $V_{\mathrm{BC}}$ is the volume of the blood cell, and $H$ is the applied magnetic field.

Additionally, the orientation of an erythrocyte in an external magnetic field is dependent on the oxygenation state of the RBC's haemoglobin, the iron-containing oxygen carrying component of RBCs $[3,4]$. In its oxygenated state, haemoglobin acts as a diamagnetic particle, and in its deoxygenated state, haemoglobin acts as a paramagnetic particle. These differing erythrocytes magnetic susceptibilities suggest that blood oxygenation affects the orientation and aggregation of flowing erythrocytes under a magnetic field and thus affects blood viscosity $[4,5]$. The arterial blood has oxygenated and deoxygenated states. The oxygenated state refers to oxygen bound to haemoglobin of erythrocytes $\left(\mathrm{HbO}_{2}\right)$ and deoxygenated state having haemoglobin lacking oxygen $(\mathrm{Hb})$.

Electrohydraulic discharges (EHDs) are produced during the rapid release of stored electrical charge across electrodes 


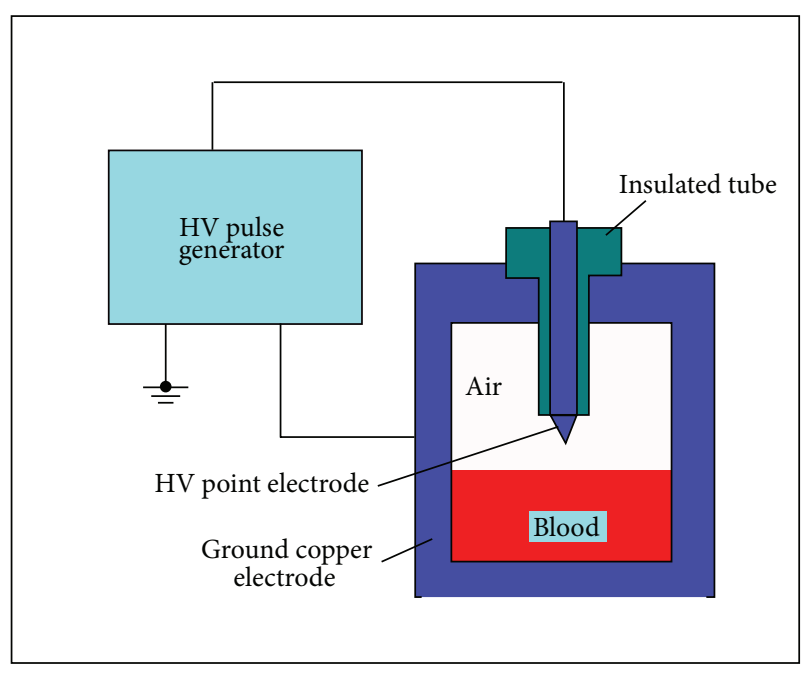

Figure 1: Schematic diagram of the electrohydraulic discharge device.

submerged below the blood surface. The resulting formation of an electrical arc across the spark gap produces a localized plasma region (i.e., ionized gas) that emits UV radiation and generates pressure and thermal shocks [6]. These phenomena can also produce radical species and ionic reactions.

One acoustic mechanism that can cause haemolysis is cavitation, which is the expansion and compression of gas bubbles caused by the applied acoustic field. The violent implosion of a bubble can lead to the production of shock waves, high-velocity liquid jets, free radical species, and strong shear forces that can damage blood cells $[7,8]$.

\section{Materials and Methods}

Before experiments were started, human blood was drawn from healthy donors and was anticoagulated with ethylene diamine tetra-acetic acid (EDTA). All experiments used whole blood. At least three $5 \mathrm{~cm}^{3}$ samples of blood were used for each exposure condition. After exposure, plasma viscosity was measured using microviscometer. The total haemoglobin level in unexposed blood and the red cell, white cell, and platelet counts in exposed and unexposed samples were measured. Schematic diagram of the electrohydraulic discharge device is shown in Figure 1.

The point electrode was made from a sharpened copper rod, which was almost totally insulated from surrounding blood and ground copper electrode (reactor vessel) by an insulated tube. A pulsed high voltage applied to the point electrode was provided by a pulse power supply (HV). It consists of a variable voltage $0-40 \mathrm{kV}$ DC source, a low inductance storage capacitor, and a rotating spark gap. The temporal evolution of the gap voltage and electrical current through the reactor were measured by a home-made resistive voltage divider and a Rogowski coil, respectively, and recorded simultaneously by the four-channel LeCroy digital oscilloscope with a sampling rate of up to $200 \mathrm{MSa} \mathrm{s}^{-1}$.

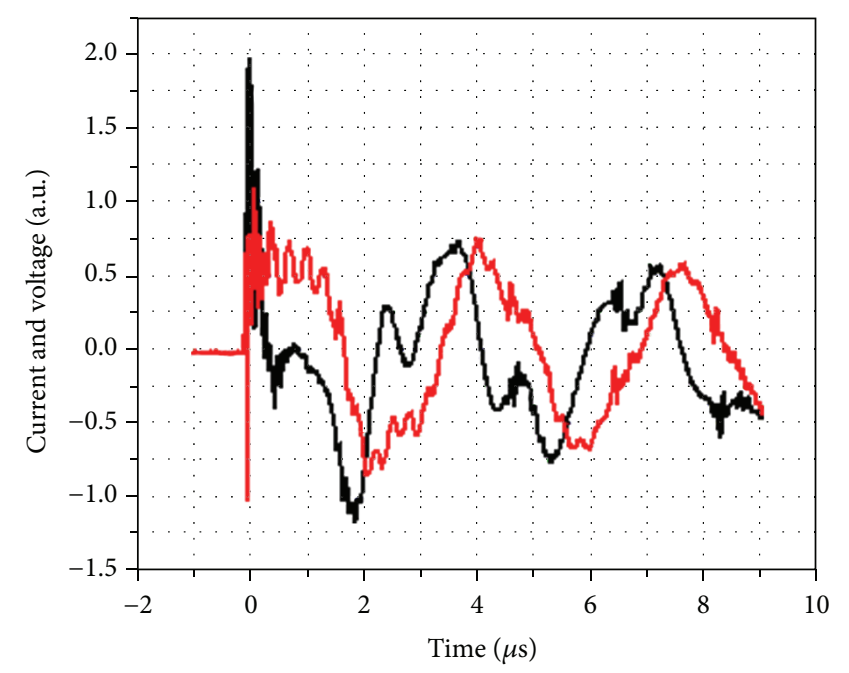

FIGURE 2: The current (red line) and voltage (black line) waveforms of the EHD device.

The blood sample was injected into the treatment chamber after cleaning and sterilizing it for the purpose of treatment. One of the electrodes was connected to the high voltage pulse generator and the other electrode was connected to the ground. The high voltage pulse generator parameters were adjusted as follows: output voltage variable from $20 \mathrm{kV}$ up to $30 \mathrm{kV}$, discharging frequency $25 \mathrm{~Hz}$, and action time variable from 60 seconds up to 210 seconds.

After treatment, the sample was taken out from the treatment chamber and put in a sterile tube after the voltage drops to zero. The blood samples were divided into two groups: one unexposed control group and test group which was exposed to a number of pulses. At the end of exposure time, the blood samples collected were analyzed in laboratory.

The circuit of the pulse voltage generator used in this study consisted of the pulse-forming capacitor $(20 \mathrm{nF})$, charged through a 50-kilo-ohm resistor by a negative DC high voltage power supply and rotating spark gap switch. As the voltage on the capacitor reached the spark-over voltage of the spark gap electrode, the capacitor was discharged, producing narrow positive high voltage pulse.

\section{Results and Discussion}

The peak value of the discharge current was approximately 450 A during the pulse. Figure 2 shows the current and voltage waveforms that characterized the EHD device. Current and voltage were measured as a function of time at an input energy of $9 \mathrm{~J}$ (maximum applied voltage $30 \mathrm{kV}$ ) and air pressure of 1 bar. From these measurements the power transfer to the reactor vessel was obtained as a function of time as shown in Figure 3.

Fourier transformation shows the amplitude spectrum of the discharge current that is displayed at the different frequency components in the pulse (Figure 4). As observed, most of the energy in the discharge current is between $100 \mathrm{kHz}$ and $50 \mathrm{MHz}$. The spectrum has several peaks 


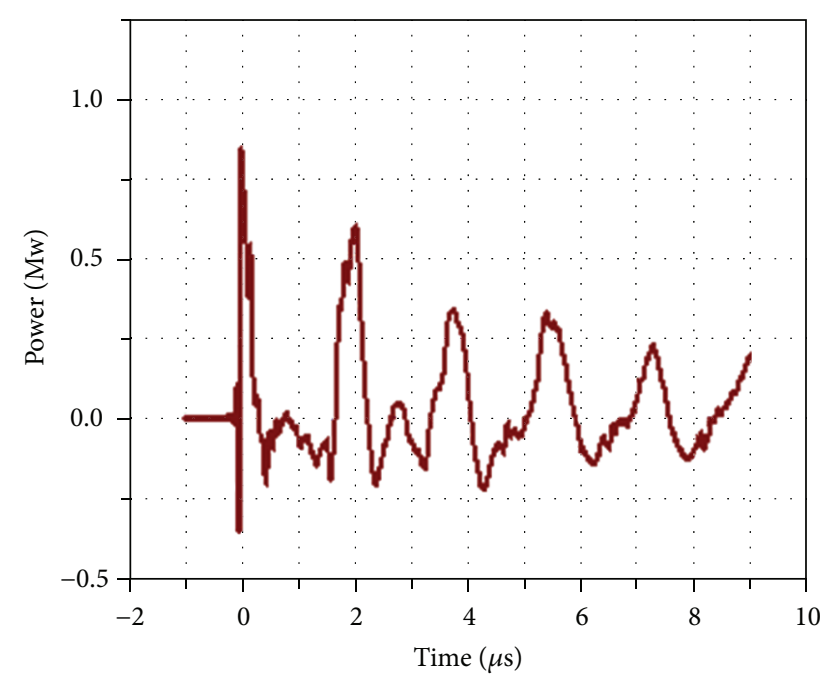

FIgURE 3: An instantaneous power waveform.

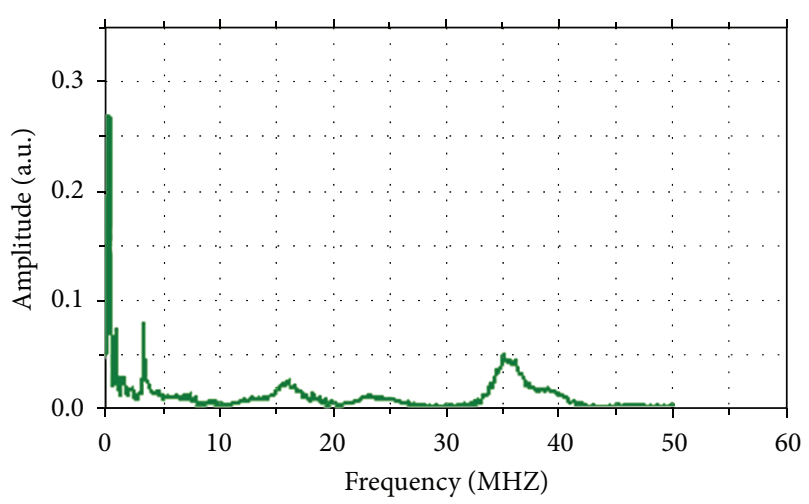

FIGURE 4: Fourier transformation of the discharge current pulse.

at different frequencies, with the maximum component at $0.29 \mathrm{MHz}$ (fundamental frequency) and the other components at $3.32 \mathrm{MHz}, 16.0 \mathrm{MHz}$, and $35.0 \mathrm{MHz}$ (harmonic frequencies). It is clear that the energy is spread over a very large frequency range, which is a characteristic feature of a short pulse.

Figure 5 indicates the measured whole blood viscosity at different exposure durations (to plasma products). Figure 6 shows the ratio of the blood viscosity under the influence of magnetic field $\eta_{\text {mag }}$ to the viscosity in the absence of magnetic field $\eta$ at different values of applied magnetic field $H$ (the exposure time being about 60 seconds).

The energy at the reactor is generally dissipated from the arc channel by radiation, shockwaves, and thermal conduction to the surrounding blood. The shockwave is the most effective parameter on hemolysis in whole blood. The results of the arc discharge reveal information about the highly ionized, high pressure, and gaseous plasma, which tends to expand in volume. The surrounding blood resists the plasma expansion; therefore, it creates high transient pressure from each discharge, resulting in the production of a shockwave

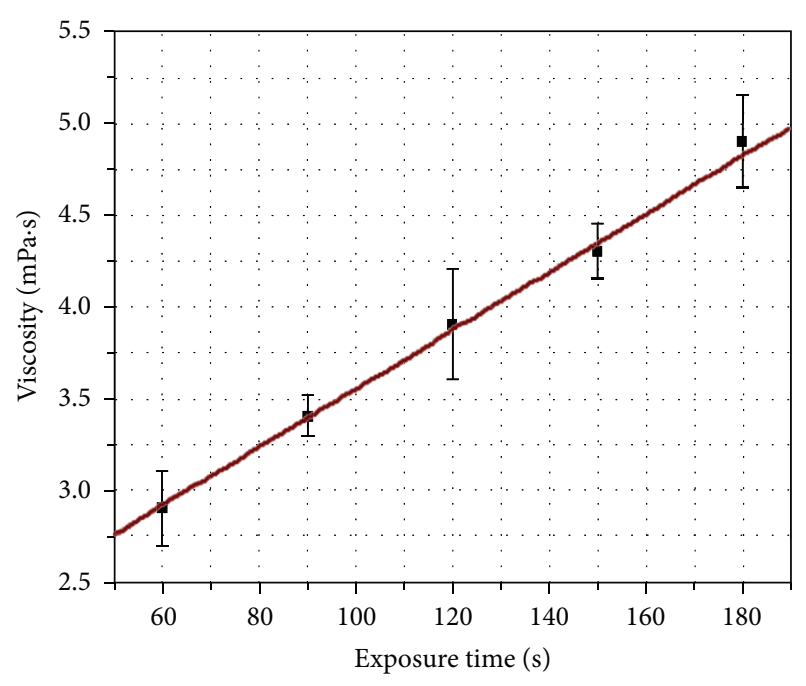

FIGURE 5: Relationship between whole blood viscosity and exposure duration.

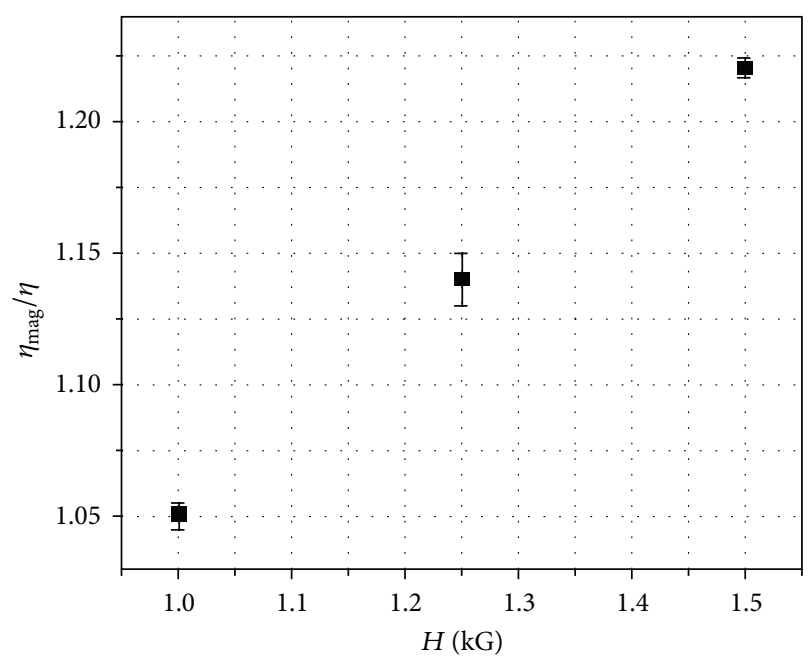

FIgURE 6: The ratio of blood viscosity under the influence of magnetic field $\eta_{\text {mag }}$ to the viscosity in the absence of magnetic field $\eta$ as a function of applied magnetic field $H$ (exposure time about 60 seconds).

[9]. It is observed that shockwave intensity is mostly related to the pulse energy.

Shockwave intensity is also a function of pressure. When the arc discharge occurs, pressure can be expressed as a function of the dissipated pulse energy [10]; that is,

$$
P_{\max } \approx \frac{335 E^{0.333}}{r}
$$

where $P$ is pressure (atm), $E$ is dissipated energy of the capacitor $(J)$, and $r$ is distance from the discharge source $(\mathrm{cm})$. The pressure $P$ is related to the shockwave velocity by

$$
\begin{gathered}
P=\mu c \rho, \\
V_{S}=\mu+c,
\end{gathered}
$$


TABLE 1: The theoretical value of pressure by the discharge energy.

\begin{tabular}{llccr}
\hline & & $20 \mathrm{kV}, 20 \mathrm{nF}, 4 \mathrm{~J}$ & $25 \mathrm{kV}, 20 \mathrm{nF}, 6.5 \mathrm{~J}$ & $30 \mathrm{kV}, 20 \mathrm{nF}, 9 \mathrm{~J}$ \\
\hline$P_{\max }$ & $R=1 \mathrm{~cm}$ & $540 \mathrm{~atm}$ & $616.6 \mathrm{~atm}$ & $696.3 \mathrm{~atm}$ \\
& $R=2 \mathrm{~cm}$ & $266 \mathrm{~atm}$ & $308.35 \mathrm{~atm}$ & $348.2 \mathrm{~atm}$ \\
\hline & $R=3 \mathrm{~cm}$ & $177 \mathrm{~atm}$ & $205.6 \mathrm{~atm}$ & $232.1 \mathrm{~atm}$ \\
$V_{s}$ & $R=1 \mathrm{~cm}$ & $1615.7 \mathrm{~cm} / \mathrm{sec}$ & $1620.75 \mathrm{~cm} / \mathrm{sec}$ & $1625.5 \mathrm{~cm} / \mathrm{sec}$ \\
& $R=2 \mathrm{~cm}$ & $1599.8 \mathrm{~cm} / \mathrm{sec}$ & $1602.38 \mathrm{~cm} / \mathrm{sec}$ & $1604.75 \mathrm{~cm} / \mathrm{sec}$ \\
& $R=3 \mathrm{~cm}$ & $1594.5 \mathrm{~cm} / \mathrm{sec}$ & $1596.256 \mathrm{~cm} / \mathrm{sec}$ & $1597.83 \mathrm{~cm} / \mathrm{sec}$ \\
\hline
\end{tabular}

where $\rho$ is density $\left(1059 \mathrm{~kg} / \mathrm{m}^{3}\right), \mu$ is particle velocity $(\mathrm{m} / \mathrm{s})$, $c$ is sound velocity ( $1584 \mathrm{~m} / \mathrm{s}$ in blood), and $V_{s}$ is shockwave velocity $(\mathrm{m} / \mathrm{s})$. The shockwave velocity is very high (over $10,000 \mathrm{~m} / \mathrm{s}$ ) at the beginning. As the shockwave propagates in blood, the shockwave velocity decreases eventually to sound velocity. Table 1 and Figure 7 show the theoretical values of pressure in terms of the discharge energy at different distances from the discharge.

Both diamagnetic and paramagnetic particles develop a magnetic moment proportional to the applied field. The magnetic moment per unit volume called the magnetic polarization, or the magnetization, is represented by the symbol $M$ in the following equation. Magnetization is the measure of how much the magnetic field affects the magnetic fluid. The simplest is the linear equation for isothermal cases $[11,12]$. Consider

$$
M=\chi H
$$

where $\chi$ is a constant called magnetic susceptibility (dimensionless number), negative for diamagnetic particles and positive for paramagnetic. The magnetic susceptibility of blood $\chi$ has already been measured to be $-6.6 * 10^{-7}$ for the oxygenated and $3.5 * 10^{-6}$ for the deoxygenated blood, respectively [13]. The oxygenated erythrocytes have diamagnetic properties because of the $S=0$ spin state of the oxyhaemoglobin. The ferrous deoxyhaemoglobin with its $S=$ 2 spin state is responsible for the paramagnetic behavior of the deoxygenated erythrocytes. All other erythrocytes are paramagnetic with different susceptibility depending on the spin state of the methemoglobin, high at $S=5 / 2$ or low at $S=1 / 2$.

An external magnetic field consists of high voltage power supply, capacitor bank, air gap switch, and solenoid around EHD reactor as shown in Figure 8. Discharging the capacitor bank through the inductive coil, electric energy is transformed into magnetic energy and a sinus-shaped magnetic field pulse can be generated. The instantaneous field in the coil may be determined by measuring the current that circulates through the solenoid.

Typical values of the circuit parameters are $C=1 \mu \mathrm{F}$ and $V=10 \mathrm{KV}$, giving an estimated peak field of $2 \mathrm{k}$-Gauss.

When the blood is exposed to the magnetic field, the action of magnetization will introduce a rotational motion to orient the fluid particles with the magnetic field. The orientation of blood cells when subjected to a magnetic field is due to the magnetic torque $\tau$. The magnetic torque $\tau$ is due to the difference in the orientation between the

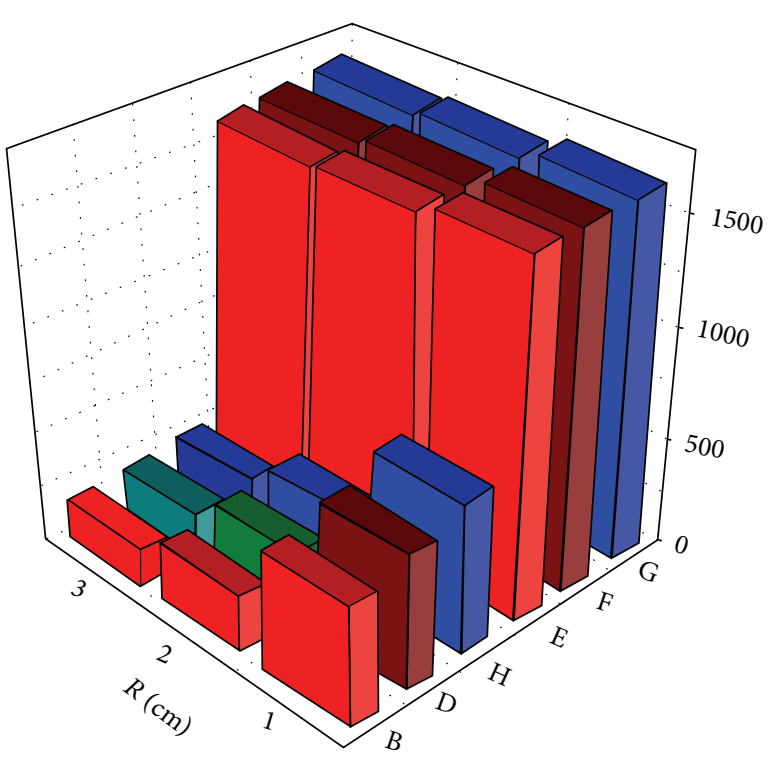

FIGURE 7: The theoretical value of pressure as a function of discharge energy. B, D, and $H$ are the pressures (atm) and $\mathrm{E}, \mathrm{F}$, and $\mathrm{G}$ are the shock velocities $(\mathrm{cm} / \mathrm{s})$ at different energies $4 \mathrm{~J}, 6.5 \mathrm{~J}$, and $9 \mathrm{~J}$, respectively, at different distances $R(\mathrm{~cm})$ from the discharge.

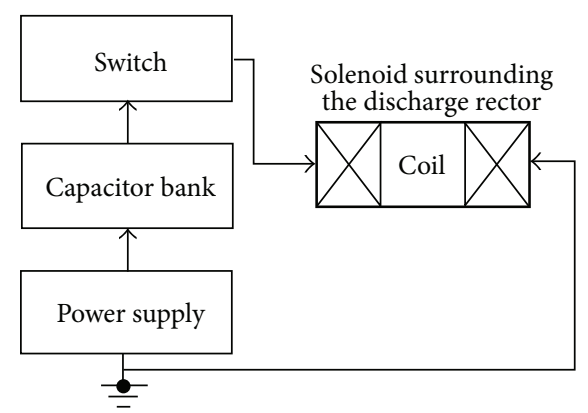

FIGURE 8: Schematic representation of pulsed magnetic field generator.

magnetization vector and the applied field. When the blood cells are suspended in the plasma without applied field, it has a viscosity $\eta$. However, if the magnetic field is applied, then the blood cells and the surrounding plasma fluid will interact and, combined with the magnetic force, increase the viscosity of the blood. An increase of the viscosity due to the magnetic torques exerted on the blood cells, which in turn increase the frictional coupling between the plasma fluid and blood cells. 
The voltage pulsation introduces electric field, and temperature jump at the same time leads to haemolysis of the blood cells. The haemolysis occurs because of the osmotic imbalance generated by the leakage of ions and small molecules. The magnitude of the temperature jump depends on the square of current and duration of the pulse. Also, the cavitation which is expansion and compression of gas bubbles caused by shock wave that can damage the blood cells. Ultraviolet radiation may cause ultrastructure changes in the platelet membrane. The ultraviolet light increases the ion permeability of human red blood cell membranes. Therefore, such ultraviolet radiation may initiate aggregation by direct effect on blood cells. The sonochemical effects are due to the electrohydraulic cavitation in the blood that causes the formation of cavitation bubbles which can grow and implode under the variations of the pressure field of the ultrasonic waves. In the case of blood structured with air, these conditions give rise to cleavage of blood and direct destruction of the suspended cells.

\section{References}

[1] G. D. O. Lowe, "Blood rheology in vitro and in vivo," Baillière's Clinical Haematology, vol. 1, no. 3, pp. 597-5636, 1987.

[2] T. Higashi, A. Yamagishi, T. Takeuchi et al., "Orientation of erythrocytes in a strong static magnetic field," Blood, vol. 82, no. 4, pp. 1328-1334, 1993.

[3] K. H. Man and A. Bruno Frazier, "Continuous magnetophoretic separation of blood cells in microdevlce format," Journal of Applied Physics, vol. 96, no. 10, pp. 5797-5802, 2004.

[4] T. Yamamoto, Y. Nagayama, and M. Tamura, "A blood-oxygenation-dependent increase in blood viscosity due to a static magnetic field," Physics in Medicine and Biology, vol. 49, no. 14, pp. 3267-3277, 2004.

[5] M. Okazaki, N. Maeda, and T. Shiga, "Effects of an inhomogeneous magnetic field on flowing erythrocytes," European Biophysics Journal, vol. 14, no. 3, pp. 139-145, 1987.

[6] W. K. Ching, A. J. Colussi, H. J. Sun, K. H. Nealson, and M. R. Hoffmann, "Escherichia coli disinfection by electrohydraulic discharges," Environmental Science and Technology, vol. 35, no. 20, pp. 4139-4144, 2001.

[7] D. L. Miller, "The influence of hematocrit on hemolysis by ultrasonically activated gas-filled micropores," Ultrasound in Medicine and Biology, vol. 14, no. 4, pp. 293-297, 1988.

[8] D. L. Miller and R. M. Thomas, "A comparison of hemolytic and sonochemical activity of ultrasonic cavitation in a rotating tube," Ultrasound in Medicine and Biology, vol. 19, no. 1, pp. 8390, 1993.

[9] H. Y. Lee, H. S. Uhm, H. N. Choi, Y. J. Jung, B. K. Kang, and H. C. Yoo, "Underwater discharge and cell destruction by shockwaves," Journal of the Korean Physical Society, vol. 42, no. 2, pp. S880-S884, 2003.

[10] F. B. A. Frugel, High Speed Pulse Technology, chapter 1, Academic Press, London, UK, 1965.

[11] Y. Haik, V. Pai, and C. J. Chen, "Apparent viscosity of human blood in a high static magnetic field," Journal of Magnetism and Magnetic Materials, vol. 225, no. 1-2, pp. 180-186, 2001.

[12] E. Rosensweig, Ferrohydrodynamics, Cambridge University Press, Cambridge, UK, 1985.
[13] E. Blums, A. Cebers, and M. M. Maiorov, Magnetic Fluids, Walter de Gruyter, Berlin, Germany, 1997. 

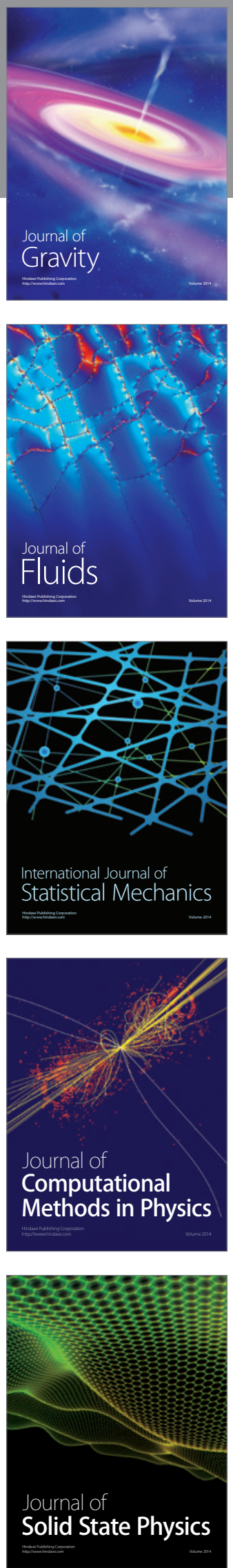

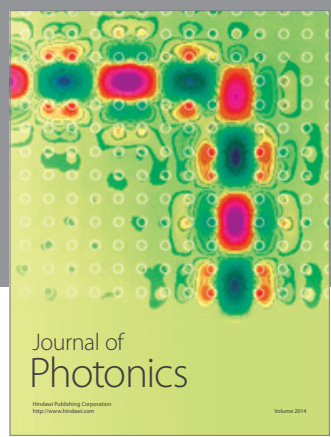

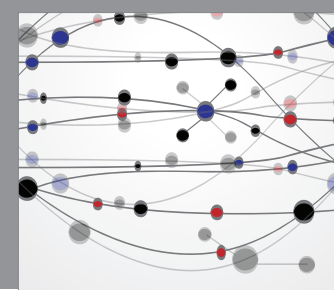

The Scientific World Journal

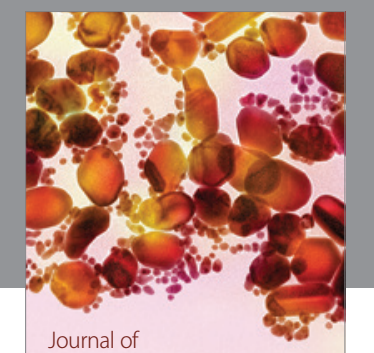

Soft Matter
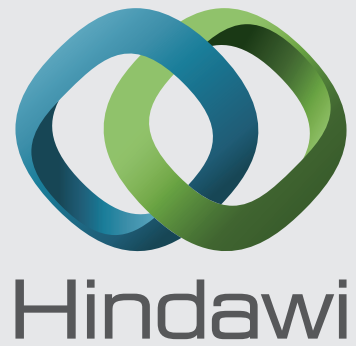

Submit your manuscripts at

http://www.hindawi.com
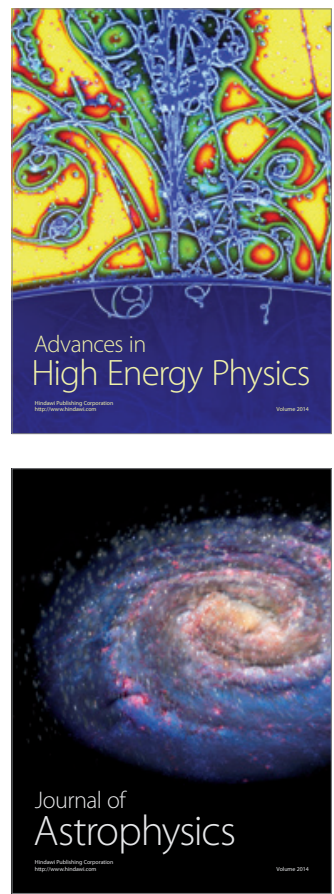
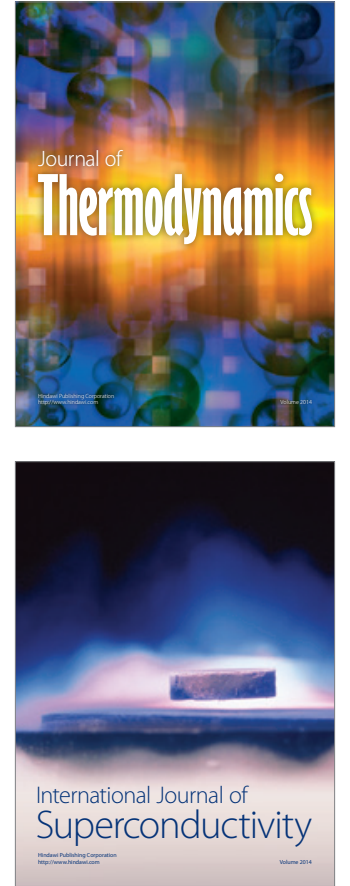
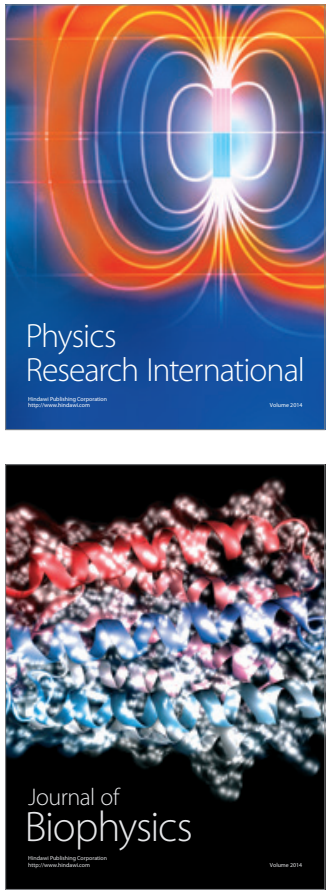
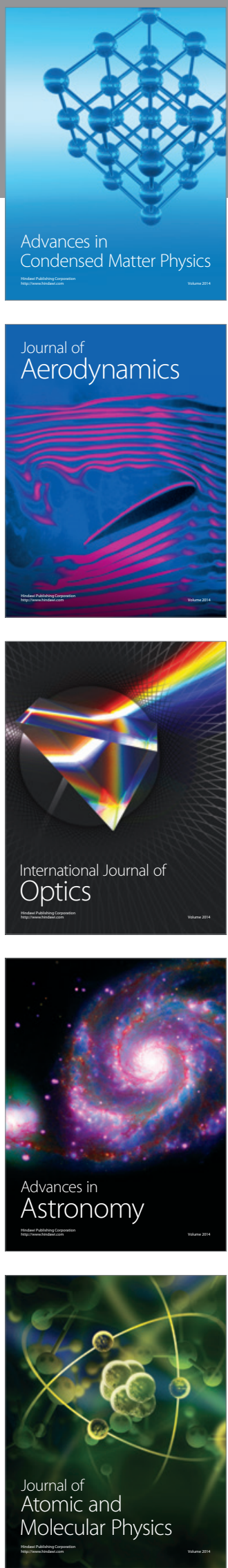